\title{
Construção e Validação de um Sistema Integrado de Dados de Intervenção Coronária Percutânea no Brasil (Registro ICP-BR): Perfil Clínico dos Primeiros 1.249 Pacientes Incluídos
}

\author{
Leonardo J. C. de Paula1, Pedro A. Lemos' ${ }^{1}$, Cesar Rocha Medeiros², José A. Marin-Neto ${ }^{3}$, \\ Geraldo Luiz Figueiredo ${ }^{3}$, Carisi A. Polanczyk ${ }^{4}$, Marco V. Wainstein ${ }^{4}$, Antônio L. P. Ribeiro ${ }^{5}$, \\ Lucas Lodi-Junqueira ${ }^{5}$, Flavio R. A. Oliveira ${ }^{6}$, Rogério Sarmento-Leite ${ }^{7}$, Luiz Alberto Mattos ${ }^{8}$, \\ Marcelo J. C. Cantarelli ${ }^{9}$, Fábio Sândoli de Brito Jr. ${ }^{9}$, Antonio C. C. Carvalho ${ }^{9}$, Maurício R. Barbosa ${ }^{9}$
}

\section{RESUMO}

Introdução: A intervenção coronária percutânea cresceu de modo expressivo nas últimas décadas. Entretanto, relatos de resultados imediatos e a longo prazo desse procedimento em instituições brasileiras são esporádicos e restritos a alguns centros. A presente proposta objetiva descrever um sistema nacional para a avaliação dos indivíduos tratados por intervenção coronária percutânea no Brasil. Método: O Registro ICP-BR foi constituído por meio de rede informatizada para a captação de dados, via web, sobre angioplastias coronárias realizadas no dia a dia da cardiologia intervencionista, sem critérios de exclusão. Em sua fase piloto, 8 centros nacionais foram selecionados para a coleta inicial de dados. Relatamos o perfil clínico e a evolução intra-hospitalar dos primeiros pacientes incluídos. Resultados: De março de 2009 a dezembro de 2009, foram incluídos 1.249 pacientes na base de dados. No total, $60 \%$ foram tratados pelo Sistema Único de Saúde,

\section{ABSTRACT}

Construction and Validation of an Integrated Percutaneous Coronary Intervention Data System in Brazil (ICP-BR Registry): Clinical Profile of the

First 1,249 Patients Included

Background: Percutaneous coronary intervention has grown dramatically in recent decades. However, reports of immediate and long-term results of this procedure in Brazilian institutions are sporadic and limited to some centers. This study is aimed at describing a national system to evaluate patients treated by percutaneous coronary intervention in Brazil. Method: The ICP-BR Registry was established by a computerized network for data capture on coronary angioplasties performed in day-to-day interventional cardiology, without exclusion criteria. In the pilot phase 8 national centers were selected for the initial data collection. We report the clinical profile and in-hospital evolution of the

\footnotetext{
1 Instituto do Coração do Hospital das Clínicas da Faculdade de Medicina da Universidade de São Paulo (InCor/HCFMUSP) - São Paulo, SP, Brasil.

2 Rede D'Or de Hospitais - Rio de Janeiro, RJ, Brasil.

3 Hospital de Clínicas da Faculdade de Medicina da Universidade de São Paulo - Ribeirão Preto, SP, Brasil.

${ }^{4}$ Hospital de Clínicas da Universidade Federal do Rio Grande do Sul (UFRGS) - Porto Alegre, RS, Brasil.

${ }^{5}$ Hospital das Clínicas da Universidade Federal de Minas Gerais (UFMG) - Belo Horizonte, MG, Brasil.

${ }^{6}$ Instituto de Medicina Integral Professor Fernando Figueira (IMIP) Recife, PE, Brasil.

7 Instituto de Cardiologia - Fundação Universitária de Cardiologia (IC-FUC) - Porto Alegre, RS, Brasil.

${ }^{8}$ Instituto Dante Pazzanese de Cardiologia (IDPC) - São Paulo, SP, Brasil.

${ }^{9}$ Sociedade Brasileira de Hemodinâmica e Cardiologia Intervencionista (SBHCl) - São Paulo, SP, Brasil.

Correspondência: Pedro A. Lemos. Serviço de Hemodinâmica InCor/HCFMUSP. Av. Dr. Enéas Carvalho de Aguiar, 44 - Cerqueira

César - São Paulo, SP, Brasil - CEP 05403-000

E-mail: pedro.lemos@incor.usp.br

Recebido em: 25/8/2010 • Aceito em: 5/9/2010
} 
Paula LJC, et al. Construção e Validação de um Sistema Integrado de Dados de Intervenção Coronária Percutânea no Brasil (Registro ICP-BR): Perfil Clínico dos Primeiros 1.249 Pacientes Incluídos. Rev Bras Cardiol Invasiva. 2010;18(3):256-62.

$38 \%$ por planos de saúde e $2 \%$ eram pagantes. A média de idade era de $63,7 \pm 11,3$ anos, $36 \%$ eram diabéticos, $12 \%$ tinham cirurgia prévia e $27 \%$ tinham angioplastia prévia. À admissão, 39\% eram estáveis e 18\% tinham infarto com supradesnivelamento do segmento ST. Ultrassom intracoronário ou reserva fracionada de fluxo foram utilizados em 2,8\% dos casos. Stents foram utilizados em 93\% dos procedimentos, sendo farmacológicos em $16,2 \%$ dos pacientes. A mortalidade foi de $0,2 \%$ nos pacientes estáveis, de $2,4 \%$ nos casos de coronariopatia aguda sem supradesnivelamento do segmento ST, de $6,1 \%$ nos pacientes com infarto com supradesnivelamento do segmento ST e de 3,6\% naqueles com "equivalente anginoso". Conclusão: Descrevemos a construção e a efetiva implementação de sistema informatizado para a coleta detalhada de dados sobre procedimentos de intervenção percutânea coronária no Brasil. Em decorrência do caráter inclusivo sem restrições (all-comers) e do seguimento prospectivamente desenhado dos pacientes, esse sistema de captação e registro poderá contribuir de forma decisiva para que se trace o perfil das intervenções percutâneas coronárias em nosso País.

DESCRITORES: Angioplastia. Angioplastia transluminal percutânea coronária. Perfil de saúde. Evolução clínica. Sistemas de informação.

A intervenção coronária percutânea é hoje a modalidade de revascularização mais comumente empregada no tratamento da doença arterial coronária, associando-se à melhora da qualidade de vida e à redução da morbidade e da mortalidade, especialmente em pacientes de maior risco. Em nosso meio, a intervenção coronária percutânea é largamente utilizada tanto no âmbito do Sistema Único de Saúde (SUS) quanto no contexto da Saúde Suplementar.

É sabido que os resultados precoces e tardios da intervenção coronária percutânea são fortemente modulados pelas características clínicas e anatômicas dos indivíduos tratados, bem como por especificidades relacionadas aos serviços de atendimento ao paciente. ${ }^{1-4}$ É importante assinalar que tanto o perfil das populações de interesse quanto as peculiaridades dos sistemas de saúde são dependentes de fatores regionais. ${ }^{5,6}$ Observa-se que as informações relativas ao desempenho clínico e aos custos envolvidos com procedimentos de alta complexidade em uma determinada realidade não são necessariamente extrapoláveis a outros contextos socioeconômicos. .,6,7 $^{4}$

A despeito do conhecimento do impacto clínico da intervenção coronária percutânea a curto, médio e longo prazos, a maior parte da informação científica é baseada na experiência de centros estrangeiros, disponível na literatura médica. Portanto, convém assinalar que as informações advindas de outros países podem não refletir a realidade brasileira, tornando-se imperativa a coleta de dados próprios dos centros brasileiros. first patients included. Results: From March 2009 to December 2009, 1,249 patients were included in the database. In total, $60 \%$ were treated by the Unified Health System, $38 \%$ by health insurance plans and $2 \%$ were private patients. Mean age was $63.7+11.3$ years, $36 \%$ were diabetic, $12 \%$ had prior surgery and $27 \%$ prior angioplasty. Upon admission, $39 \%$ were stable and $18 \%$ had ST elevation myocardial infarction. Intracoronary ultrasound or fractional flow reserve was performed in $2.8 \%$ cases. Stents were used in $93 \%$ of procedures, and drug-eluting stents in $16.2 \%$ of the patients. Mortality was $0.2 \%$ in stable patients, $2.4 \%$ in patients with acute coronary syndromes without ST elevation, 6.1\% in patients with ST elevation myocardial infarction and $3.6 \%$ in those with anginal equivalent. Conclusion: We describe the development and implementation of a computerized system to collect detailed data on percutaneous coronary intervention procedures in Brazil. Given the inclusive unrestricted character (all-comers) and prospective follow-up of patients, this data capture and recording system may contribute decisively to profile percutaneous coronary intervention in our country.

KEY-WORDS: Angioplasty. Angioplasty, transluminal, percutaneous coronary. Health profile. Clinical evolution. Information systems.

A necessidade de dados nacionais fidedignos implica a realização de estudos populacionais, com número significativo de indivíduos incluídos em ambiente multicêntrico de investigação científica rigoroso com coleta prospectiva de dados. O Registro CENIC (Central Nacional de Intervenções Cardiovasculares) da Sociedade Brasileira de Hemodinâmica e Cardiologia Intervencionista, instituído a partir da década de 1990, coleta informações sobre intervenções coronárias percutâneas realizadas em diversos centros no território nacional mediante envio espontâneo e voluntário de dados por parte dos operadores. ${ }^{8}$ A despeito de suas inegáveis qualidades e consequentes contribuições quanto ao conhecimento da intervenção coronária percutânea realizada no Brasil, deve-se reconhecer que os dados da CENIC são limitados aos pacientes incluídos de forma livre (sem se ter controle quanto aos critérios pelos quais os centros incluem ou deixam de incluir), e que se referem exclusivamente ao período periprocedimento, sem dados evolutivos. Portanto, ainda hoje não detemos no País um sistema de informação de larga escala com o detalhamento clínico e técnico sobre os procedimentos coronários percutâneos realizados no Brasil, vistos globalmente por amostras sem restrição nos vários centros, juntamente com a subsequente evolução precoce e tardia dos indivíduos tratados.

É a partir da necessidade de dados nacionais com aquelas características que nasceu a presente proposta, a qual objetiva a formação de sistema que possibilite a coleta pormenorizada de dados globais relativos às características clínicas e angiográficas e à evolução 
dos indivíduos tratados por intervenção coronária percutânea no Brasil. Este relato objetiva detalhar, pela primeira vez, as bases técnicas do banco de dados construído bem como o perfil clínico e a evolução intrahospitalar dos primeiros pacientes incluídos na fase piloto de instalação deste registro nacional.

\section{MÉTODOS}

A presente plataforma de coleta de dados objetivava registrar os perfis clínicos e a evolução dos procedimentos, basal e pós-tratamento, de indivíduos com doença coronária regularmente tratados nas instituições envolvidas. Este projeto não previa, bem como não implicou, qualquer modificação e/ou interferência na conduta clínica individual, mas somente na coleta de dados de maneira parametrizada e hierarquizada com o intuito de descrever as características e resultados da intervenção coronária percutânea inerentes a um determinado número de pacientes.

\section{Financiamento}

Este projeto, intitulado "Construção e implementação de sistema para a integração e gestão de dados da intervenção coronária percutânea (ICP) no Brasil: Projeto piloto", foi subvencionado por meio do edital no 33 / 2007 do Ministério da Ciência e Tecnologia (MCT)/Conselho Nacional de Desenvolvimento Científico e Tecnológico (CNPq)/Secretaria de Ciência, Tecnologia e Insumos Estratégicos (SCTIE) e Departamento de Ciência e Tecnologia (DECIT) do Ministério da Saúde (MS).

\section{Construção da base de dados}

1) Infraestrutura instalada e software operacional

Criou-se base de dados acessível de maneira remota, via web, através de "login" e "senha" cadastrados de acordo com hierarquia de privilégios para os diversos usuários. O acesso era obtido através do website http:// www.registro-icp-brasil.com (porta de acesso disponível 24 horas por dia, 7 dias por semana) registrado com o provedor de domínios "e-dominios", que definia a primeira rota de acesso, redirecionando para o provedor de acesso "superdns", que, por sua vez, redirecionava o acesso ao IP (Internet Protocol) onde se encontra instalado o servidor de aplicação que gerenciava o sistema e o acesso ao banco de dados através do IIS (Internet Information Service) no Windows XP Professional.

No-breaks foram utilizados para garantir o fornecimento contínuo de energia. A temperatura foi controlada com ar condicionado e racks, com ventiladores para melhor escoamento de calor. Servidores interligados entre si estiveram conectados ao backbone da Embratel via gigabit Ethernet, garantindo um link de uso exclusivo com 2.000 Mbps FULL (Download/Upload). Os servidores utilizados foram dois computadores dedicados (para melhor desempenho e segurança de acesso), instalados em DataCenter, sendo um servidor de aplicação (S.O.
Windows XP Professional) e outro servidor de banco de dados (S.O. Linux; B.D. Oracle 10g). Neste modelo de acesso, estima-se uma disponibilidade de 99,5\% do servidor (hardware) e do ambiente (Sistema Operacional) (Figura 1). A equipe utilizou a ferramenta Delphi IntraWeb, considerando a facilidade de uso, a segurança e a experiência da equipe com essa modalidade de desenvolvimento por mais de cinco anos.

2) Base de dados: tabelas e campos

O sistema foi desenvolvido e testado por meio da inclusão, sem restrições ou critérios de exclusão, de casos reais sucessivos, realizados nas instituições participantes, conforme descrito a seguir:

- dados demográficos;

- fatores de risco cardiovascular;

- antecedentes cardíacos;

- comorbidades;

- quadro clínico e indicação do procedimento;

- via de acesso, materiais e farmacologia adjunta;

- características angiográficas da lesão ou das lesões;

- estratégia terapêutica intervencionista (técnicas e materiais especiais);

- evolução intra-hospitalar;

- evolução pós-alta hospitalar; e

- terapêutica farmacológica adjunta.

A Figura 2 ilustra as relações entre as diversas planilhas constituintes da base de dados.

3) População e seguimento clínico

Após a construção e a estruturação da base de dados, passou-se à inclusão, sem restrições e simultanea-

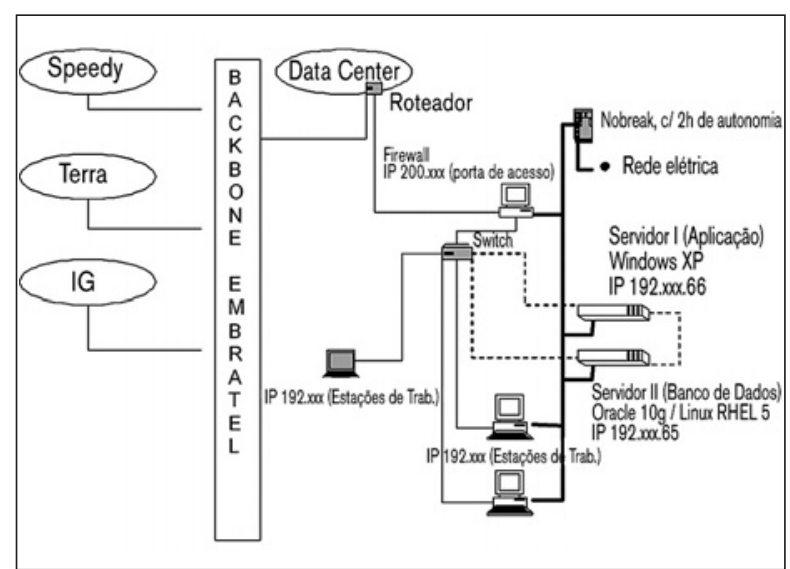

Figura 1 - Esquema da infraestrutura instalada para o funcionamento computadorizado da base de dados. $\mathbb{I P}=$ Internet Protocol; trab. = trabalho. 
Paula LJC, et al. Construção e Validação de um Sistema Integrado de Dados de Intervenção Coronária Percutânea no Brasil (Registro ICP-BR): Perfil Clínico dos Primeiros 1.249 Pacientes Incluídos. Rev Bras Cardiol Invasiva. 2010;18(3):256-62.

mente nos vários centros, de maneira prospectiva, de uma população de 1.249 indivíduos. A inclusão desses pacientes, nessa fase piloto, teve o intuito de testar a consistência e a operacionalidade da base de dados e dos sistemas de rastreamento pós-alta.

Foram incluídos sucessivamente indivíduos tratados com intervenção coronária percutânea, com ou sem sucesso, por qualquer técnica intervencionista, nos oito centros de referência da rede de monitoramento e que concordaram em ser abordados para a coleta de suas informações de evolução clínica. Não havia critérios de exclusão para o presente estudo, exceto recusa por parte do paciente ou seus responsáveis em participar do registro.

Os oito centros de referência selecionados para a coleta inicial são: Instituto do Coração do Hospital das Clínicas da Faculdade de Medicina da Universidade de São Paulo (InCor/HCFMUSP) e Instituto Dante Pazzanese de Cardiologia (IDPC), ambos em São Paulo (SP), Hospital das Clínicas da Faculdade de Medicina de Ribeirão Preto (HCFMRP/USP), em Ribeirão Preto (SP), Hospital de Clínicas de Porto Alegre (HCPA), em Porto Alegre (RS), Hospital das Clínicas da Universidade Federal de Minas Gerais (HC-UFMG), em Belo Horizonte (MG), Rede D'Or de Hospitais, no Rio de Janeiro (RJ), Instituto de Cardiologia do Rio Grande do Sul-Fundação Universitária de Cardiologia (IC-FUC), em Porto Alegre (RS), e Instituto de Medicina Integral (IMIP), no Recife (PE).

Os dados basais, do procedimento e da evolução clínica intra-hospitalar foram coletados prospectivamente pela equipe local das instituições participantes, composta por coordenador, investigadores e bolsista supervisor (técnico em pesquisa).

\section{Considerações estatísticas e análise de dados}

O estudo apresenta uma proposta essencialmente descritiva e o cálculo do tamanho da amostra foi estimado para assegurar precisão ao redor de desfechos

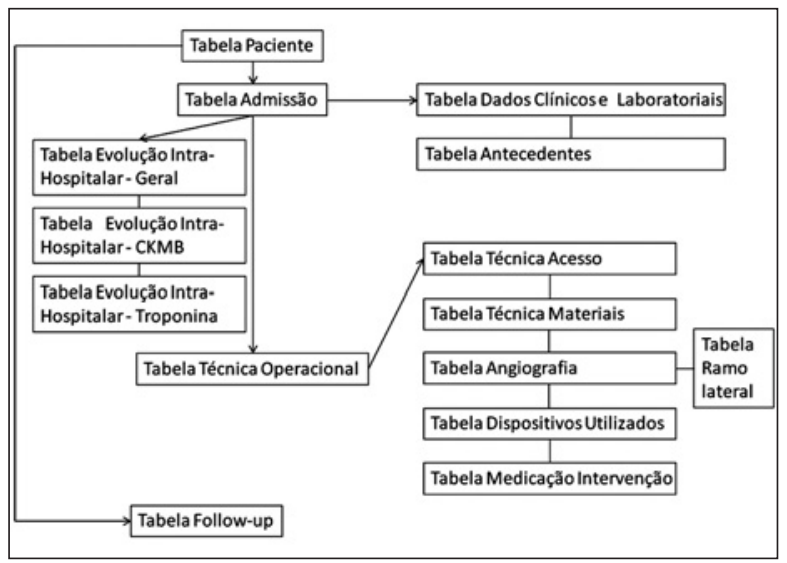

Figura 2 - Relações lógicas entre as diversas planilhas constituintes da base de dados. importantes a serem avaliados. Estimou-se que a mortalidade global após um ano de seguimento seja de 7,5\%, tendo como base dados preliminares obtidos a partir da curva de sobrevida de indivíduos tratados com intervenção coronária percutânea no InCor/HCFMUSP (perfil geral dessa população preliminar: 4.289 pacientes; idade $62 \pm 12$ anos; 66\% homens; 28\% diabéticos; $33 \%$ admitidos com infarto agudo). Dessa forma, foi calculado que um tamanho amostral de mil pacientes seria suficiente para produzir intervalo de confiança de $95 \%$ (valor da mortalidade esperada de 7,5\% \pm $1,6 \%$ ao redor do mesmo, isto é, de 5,9\% a 9,1\%). ${ }^{9-11}$ Ainda com base na população preliminar descrita, um número amostral de mil indivíduos seria suficiente para demonstrar, com poder estatístico de $\sim 100 \%$ e nível de significância de 0,05, um incremento na mortalidade em função do aumento da idade com hazard ratio de 1,34 para cada década de vida adicional (em população com desvio padrão 1,1570 décadas de vida), ajustando-se para uma mortalidade global antecipada de $7,5 \%$. Os dados foram descritos como médias, desvios padrão e porcentuais, conforme o tipo de variável.

\section{RESULTADOS}

Desde a etapa inicial de operacionalização da base, a inclusão de dados foi testada quanto a ergonomia do processo, adequação das informações coletadas e fluidez do sistema de retroalimentação entre o gerente do sistema e os usuários. O dinamismo da produção de versões para aperfeiçoamentos e certificações em torno da arquitetura da informação e consistência do input de dados no sistema foi característica fundamental para a obtenção da versão aperfeiçoada e final 1.3.02. O layout da webpage inicial de acesso à base de dados é ilustrado na Figura 3.

Os objetivos deste projeto foram atingidos a contento com o conhecimento e a gestão fidedigna de casos genuinamente brasileiros por meio da construção de sistema informatizado dotado de configuração plena ade-

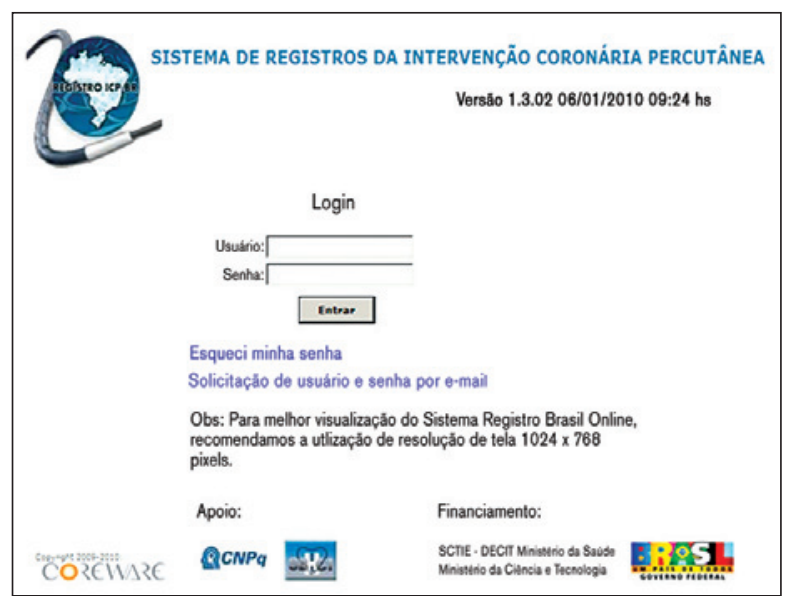

Figura 3 - Layout da webpage inicial de acesso à base de dados. 
quada à análise pormenorizada dos detalhes basais, do procedimento e da evolução desses indivíduos submetidos ao tratamento por intervenção coronária percutânea. Este sistema encontra-se pronto em esquema ininterrupto de 24 horas por dia, 7 dias por semana, é compatível com outras bases de dados existentes bem como acena para outras possibilidades de utilização em larga escala sem interferir no cotidiano da prática clínica. Dessa forma, além da obtenção de plataforma informatizada para a coleta prospectiva de dados sobre a prática e os resultados da intervenção coronária percutânea no Brasil pronta e testada para outras utilizações, os dados finais compilados permitiram analisar de maneira preliminar, porém relativamente ampla, o perfil de risco e a evolução pós-procedimento da população de pacientes com doença coronária tratados nas instituições participantes.

Os primeiros 1.249 pacientes incluídos prospectivamente na base de dados, que compõem a população deste relato, foram submetidos a intervenção coronária percutânea no período entre março de 2009 e dezembro de 2009. Contribuíram para a inclusão de pacientes, nessa fase inicial, seis dos oito centros de referência selecionados: InCor/HCFMUSP ( $\mathrm{n}=573$ ), Rede D'Or ( $n=373)$, HCFMRP/USP ( $n=185)$, HCUFMG ( $n=55)$, IMIP $(n=38)$ e IC-FUC $(n=25)$.

O perfil clínico basal dos pacientes é apresentado na Tabela 1. A média de idade à admissão foi de $63,7 \pm$ 11,3 anos e a maioria era do sexo masculino $(67,1 \%)$. Do total de pacientes atendidos, 59,8\% foram tratados pelo SUS, 38,4\% foram tratados por planos de saúde e 1,8\% eram pagantes. Quanto às características clínicas, $36,1 \%$ dos pacientes eram diabéticos, $12 \%$ foram submetidos a cirurgia de revascularização cirúrgica do miocárdio previamente e $26,9 \%$ possuíam angioplastia coronária pregressa.

\section{TABELA 1 \\ Perfil clínico dos pacientes incluídos}

\begin{tabular}{lc} 
Período de inclusão & $3 / 2009-12 / 2009$ \\
Número de pacientes & 1.249 \\
Fonte de financiamento, \% & \\
$\quad$ Sistema Único de Saúde & 59,8 \\
$\quad$ Planos de saúde & 38,4 \\
$\quad$ Pagantes & 1,8 \\
Idade, anos & $63,7 \pm 11,3$ \\
Sexo masculino, \% & 67,1 \\
Diabetes, \% & 36,1 \\
Cirurgia coronária prévia, \% & 12 \\
Intervenção coronária & \\
percutânea prévia, \% & 26,9 \\
Utilização de ultrassom intracoronário & \\
ou reserva fracionada de fluxo, \% & 2,8 \\
Utilização de stent, \% & 93,2 \\
$\quad$ Utilização de stent farmacológico & 16,2 \\
\hline
\end{tabular}

Quanto ao tratamento implementado, 93,2\% dos pacientes receberam stents coronários, sendo implantados, em 16,2\% dos pacientes, stents farmacológicos. As técnicas de ultrassom intracoronário e/ou reserva de fluxo fracionada foram utilizadas em 2,8\% dos casos estudados.

Em relação ao quadro clínico à admissão, 18,1\% dos pacientes tratados apresentaram-se com infarto agudo do miocárdio com supradesnivelamento do segmento ST, enquanto 39\% apresentaram-se com coronariopatia aguda sem supradesnivelamento do segmento ST. Os casos de dor torácica atípica, pacientes com quadro de isquemia silenciosa e angina estável corresponderam a $38,5 \%$ da amostra. Já 4,4\% buscaram atendimento com quadro de "equivalente anginoso" (Figura 4).

A mortalidade hospitalar global dos pacientes incluídos foi de 2,3\%. O quadro clínico à admissão demonstrou significativo impacto diferencial sobre a mortalidade nos pacientes tratados por intervenção coronária percutânea, conforme demonstrado na Figura 5. A mortalidade foi de $0,2 \%$ nos pacientes com diagnóstico de angina estável, de 2,4\% nos casos de coronariopatia aguda sem supradesnivelamento do segmento ST, de $6,1 \%$ nos pacientes com infarto agudo do miocárdio com supradesnivelamento do segmento ST, e de 3,6\% naqueles com "equivalente anginoso".

\section{DISCUSSÃO}

No presente relato foi detalhado o desenvolvimento de sistema de coleta, não enviesada por quaisquer critérios de exclusão, de dados sobre intervenção coronária percutânea como realizada em diversos centros no Brasil, de forma a permitir a análise pormenorizada das características basais, do procedimento e da evolução de indivíduos submetidos a tal tratamento. Concomitantemente, na fase de teste da base, por meio da inclusão de aproximadamente 1.300 pacientes, foram obtidos dados iniciais que possibilitaram analisar o perfil de risco

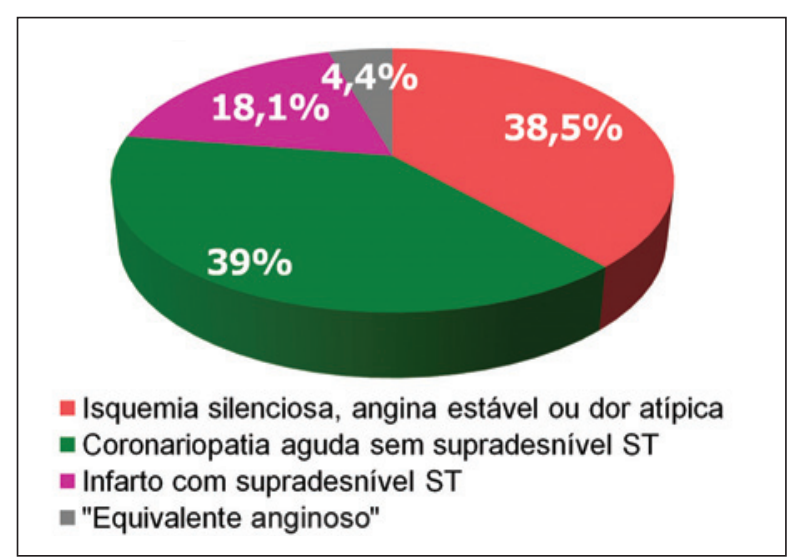

Figura 4 - Quadro clínico à admissão de pacientes tratados com intervenção coronária percutânea. 

ICP-BR): Perfil Clínico dos Primeiros 1.249 Pacientes Incluídos. Rev Bras Cardiol Invasiva. 2010;18(3):256-62.

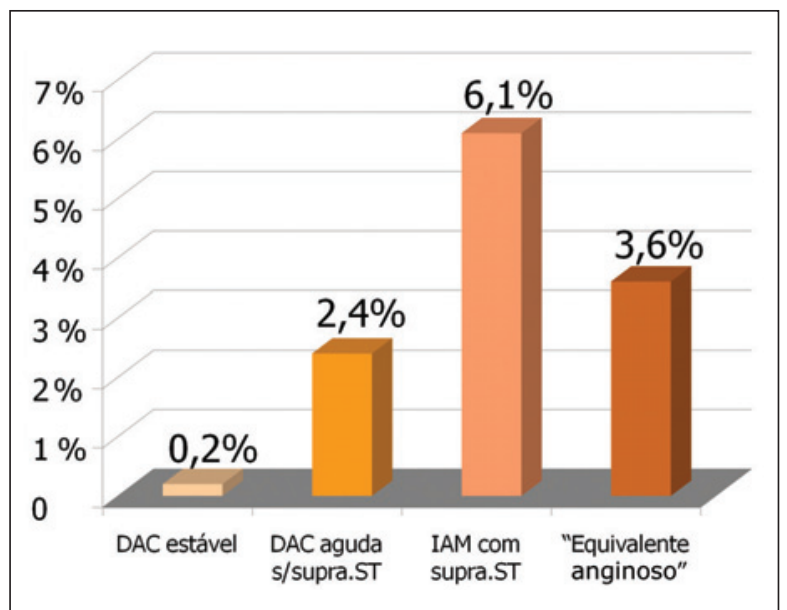

Figura 5 - Mortalidade intra-hospitalar de acordo com o quadro clínico à admissão. DAC = doença arterial coronária; IAM = infarto agudo do miocárdio; s/supra.ST = sem supradesnivelamento do segmento ST; supra.ST = supradesnivelamento do segmento ST.

de uma população de pacientes com doença coronária assim tratados e sua evolução após o procedimento.

Quanto às características clínicas, os dados evidenciam aproximadamente um terço de pacientes diabéticos, semelhante a diversos outros estudos internacionais, demonstrando a constância desse perfil de pacientes em populações tratadas por intervenção coronária percutânea. Da mesma forma, demonstramos que 12\% dos pacientes foram submetidos a revascularização miocárdica cirúrgica prévia e aproximadamente $27 \%$ a angioplastia coronária, evidenciando que quase $40 \%$ dos pacientes já haviam sido submetidos a alguma modalidade de revascularização.

É importante destacar que mais de $60 \%$ dos pacientes apresentavam quadro de síndrome isquêmica aguda à admissão, seja na forma de infarto com supradesnivelamento de ST, coronariopatia aguda sem supradesnivelamento de ST ou "equivalentes anginosos". Esses quadros demandam abordagem precoce de intervenção por possuírem risco mais elevado de complicações quando comparados a pacientes com síndromes estáveis, que corresponderam a menos de $40 \%$ da amostra, nos quais a abordagem tende a ser eletiva e com menor risco de eventos adversos. Esses dados refletem a mudança na prática intervencionista brasileira, conforme esperado pelas evidências na literatura.

Dados recentes de procedimentos realizados até 2008 e incluídos no Registro CENIC relatavam mortalidade na fase intra-hospitalar de 1,2\% em mulheres e de $0,79 \%$ em homens, ${ }^{12}$ menor que a mortalidade global de $2,3 \%$ verificada em nosso estudo. Igualmente, a mortalidade de $6,1 \%$ verificada em nosso registro para pacientes com infarto agudo do miocárdio e supradesnivelamento de ST é mais alta que a relatada na CENIC, de acordo com análise recente, a qual se situava entre $2 \%$ e 4,4\%. ${ }^{13}$ Obviamente, tais discrepâncias devem ser interpretadas considerando-se que os dados de ambos os registros não são equiparáveis em muitos aspectos, sobretudo com relação ao viés de seleção. É importante ressaltar que nosso registro incluiu casos sucessivos, não selecionados a partir de quaisquer critérios de exclusão. O perfil de risco dos pacientes refletiu-se nas taxas de mortalidade intrahospitalar encontradas. Em pacientes com síndromes estáveis, a mortalidade foi de $0,2 \%$ dos casos, porcentual bastante baixo para pacientes com coronariopatia obstrutiva com necessidade de abordagem percutânea. Mesmo em pacientes com síndromes agudas, as taxas de mortalidade encontradas foram semelhantes àquelas relatadas em controles históricos de outros países. ${ }^{14,15}$

Foi possível observar de forma contundente que o emprego de stents coronários é atualmente o método padrão para o tratamento percutâneo da coronariopatia obstrutiva, sendo utilizado em 93,2\% dos casos catalogados. No entanto, a taxa global de utilização de stents farmacológicos foi de apenas 16,2\%, índice baixo quando comparado ao observado em outras nações. ${ }^{16}$ Tal proporção refletiu o fato de $60 \%$ terem sido tratados no âmbito do SUS, que, atualmente, não prevê desembolso para a utilização desses dispositivos, bem como penetração de aproximadamente $40 \%$ de stents farmacológicos no contexto da Saúde Suplementar.

Por último, a utilização de métodos adjuntos para guiar a terapêutica percutânea ainda é um terreno pouco explorado pelos centros de hemodinâmica incluídos no estudo, apesar de serem em sua maioria centros de excelência e alta complexidade. Somente em 2,8\% dos casos foi utilizada a técnica de ultrassom intracoronário ou de medida da reserva de fluxo fracionada, demonstrando que na maioria dos casos ainda usamos exclusivamente a angiografia coronária para embasar tanto a realização do procedimento quanto a avaliação do resultado obtido após o implante do stent coronário.

\section{CONCLUSÃO}

Em concordância com o objetivo primordial deste projeto, neste relato descrevemos a construção e implementação bem-sucedida de um sistema informatizado, em rede interinstitucional, que possibilita a coleta detalhada de dados basais e da evolução de pacientes tratados com procedimentos de intervenção percutânea coronária no Brasil. Atualmente, o sistema desenvolvido encontra-se pronto para eventual agregação a bases de dados existentes. Também, os dados já coletados permitiram analisar de maneira preliminar, porém relativamente ampla, o perfil de risco e a evolução imediata pós-procedimento de população de pacientes com doença coronária tratados nas instituições participantes.

\section{CONFLITO DE INTERESSES}

Os autores declararam inexistência de conflito de interesses relacionado a este manuscrito. 


\section{REFERÊNCIAS}

1. Terkelsen CJ, Sorensen JT, Maeng M, Jensen LO, Tilsted HH, Trautner S, et al. System delay and mortality among patients with STEMI treated with primary percutaneous coronary intervention. JAMA. 2010;304(7):763-71.

2. Wu Y, Jin R, Grunkemeier GL. Validating the Clinical Outcomes Assessment Program risk model for percutaneous coronary intervention. Am Heart J. 2006;151(6): $1276-80$.

3. Good CW, Blankenship JC, Scott TD, Skelding KA, Berger PB, Wood GC. Feasibility and safety of ad hoc percutaneous coronary intervention in the modern era. J Invasive Cardiol. 2009;21(5):194-200.

4. Ide H, Mollahaliloglu S. How firms set prices for medical materials: a multi-country study. Health Policy. 2009;92(1):73-8.

5. Goodney PP, Travis LL, Malenka D, Bronner KK, Lucas FL, Cronenwett JL, et al. Regional variation in carotid artery stenting and endarterectomy in the Medicare population. Circ Cardiovasc Qual Outcomes. 2010;3(1):15-24.

6. Tiemann O. Variations in hospitalisation costs for acute myocardial infarction - a comparison across Europe. Health Econ. 2008;17(1 Suppl):S33-45.

7. Cappellaro G, Fattore G, Torbica A. Funding health technologies in decentralized systems: A comparison between Italy and Spain. Health Policy. 2009;92(2-3):313-21.

8. Sousa AG. Percutaneous cardiovascular intervention procedures in Brazil (1992-1993). Report of the National RegistryNational Center for Cardiovascular Interventions. Arq Bras Cardiol. 1994;62(4):217-23.
9. Desu MM, Raghavarao D. Sample size methodology. New York: Academic Press; 1990.

10. Hahn GJ, Meeker WQ. Statistical intervals. New York: John Wiley \& Sons; 1991.

11. Machin D, Campbell M, Fayers P, Pinol A. Sample size tables for clinical studies. Malden: Blackwell Science; 1997.

12. Lopes MACQ, Barros MAV, Oliveira IR, Martins HC, Paiva MS, Lima JAC, et al. Comparação do perfil epidemiológico, clínico e dos resultados das intervenções coronárias percutâneas entre os gêneros masculino e feminino, na população brasileira: dados do Registro CENIC. Rev Bras Cardiol Invasiva. 2008;16(4):463-73.

13. DeVito FS, Marin-Neto JA, Mattos LA. Análise comparativa da intervenção coronária percutânea com stents farmacológicos versus stents não-farmacológicos na vigência de infarto do miocárdio com supradesnivelamento do segmento ST: dados do Registro Brasileiro CENIC. Rev Bras Cardiol Invasiva. 2008; 16(4):456-62.

14. Lenzen MJ, Boersma E, Bertrand ME, Maier W, Moris C, Piscione $\mathrm{F}$, et al. Management and outcome of patients with established coronary artery disease: the Euro Heart Survey on coronary revascularization. Eur Heart J. 2005;26(12):1169-79.

15. Cook S, Walker A, Hugli O, Togni M, Meier B. Percutaneous coronary interventions in Europe: prevalence, numerical estimates, and projections based on data up to 2004. Clin Res Cardiol. 2007;96(6):375-82.

16. Hannan EL, Racz M, Holmes DR, Walford G, Sharma S, Katz S, et al. Comparison of coronary artery stenting outcomes in the eras before and after the introduction of drug-eluting stents. Circulation. 2008;117(16):2071-8. 